\title{
Evolution of Angular Momentum and Orbital Period Changes in Close Double White Dwarf Binaries
}

\author{
Seblu Humne Negu*, Solomon Belay Tessema \\ Astronomy and Astrophysics Research Development Department, Ethiopian Space Science and Technology Institute \\ (ESSTI)-Entoto Observatory and Research Center (EORC), Addis Ababa, Ethiopia \\ Email: *seblu1557@gmail.com, tessemabelay@gmail.com
}

How to cite this paper: Negu, S.H. and Tessema, S.B. (2018) Evolution of Angular Momentum and Orbital Period Changes in Close Double White Dwarf Binaries. International Journal of Astronomy and Astrophysics, 8, 275-298.

https://doi.org/10.4236/ijaa.2018.83020

Received: July 26, 2018

Accepted: September 25, 2018

Published: September 28, 2018

Copyright (c) 2018 by authors and Scientific Research Publishing Inc. This work is licensed under the Creative Commons Attribution International License (CC BY 4.0).

http://creativecommons.org/licenses/by/4.0/

\begin{abstract}
We have presented the evolution of angular momentum and orbital period changes between the component spins and the orbit in close double white dwarf binaries undergoing mass transfer through direct impact accretion over a broad range of orbital parameter space. This work improves upon similar earlier studies in a number of ways: First, we calculate self-consistently the angular momentum of the orbit at all times. This includes gravitational, tides and mass transfer effects in the orbital evolution of the component structure models, and allow the Roche lobe radius of the donor star and the rotational angular velocities of both components to vary, and account for the exchange of angular momentum between the spins of the white dwarfs and the orbit. Second, we investigate the mass transfer by modeling the ballistic motion of a point mass ejected from the center of the donor star through the inner Lagrangian point. Finally, we ensure that the angular momentum is conserved, which requires the donor star spin to vary self-consistently. With these improvements, we calculate the angular momentum and orbital period changes of the orbit and each binary component across the entire parameter space of direct impact double white dwarf binary systems. We find a significant decrease in the amount of angular momentum removed from the orbit during mass transfer, as well as cases where this process increases the angular momentum and orbital period of the orbit at the expense of the spin angular momentum of the donor and accretor. We find that our analysis yields an increase in the predicted number of stable systems compared to that in the previous studies, survive the onset of mass transfer, even if this mass transfer is initially unstable. In addition, as a consequence of the tidal coupling, systems that come into contact near the mass transfer instability boundary undergo a phase of mass transfer with their orbital period.
\end{abstract}




\section{Keywords}

Stars: DWD Binaries, Mass Transfer, Methods: Numerical

\section{Introduction}

Double white dwarf (DWD) binaries provide an interesting key to understand a variety of areas in astrophysics. Their birth properties provide insight into the evolution of their progenitors [1], as well as the dynamics of common envelope evolution [2]. From a population standpoint, the DWD binary systems may make up the largest fraction of the close binary stars in our Galaxy.

Following common envelope evolution, DWD binaries may emerge with a sufficiently short orbital period and rotational angular velocities, allowing gravitational radiation (GR), tides and mass transfer to drive the stars closer together on an astrophysically interesting time scale. Prior to the onset of mass transfer, their orbit will be shrink due to the effect of angular momentum via GR. Tidal forces are thought to synchronize the spin and orbital periods of the white dwarfs by the time that the white dwarfs are close enough together to begin transferring mass ([3] [4]).

As the degenerate components of a DWD binary orbit continues to shrink via GR, the less massive component will inevitably fill its Roche lobe and begin transferring matter to its companion and the system will enter into a stable semi-detached state. In the case where the mass transfer is stable, such systems may be identifiable as AM CVn ([5] [6]), which have extremely short orbital periods, but we still lack a uniform theoretical understanding of the detail nature of those binaries for all possible mass ratios, orbital parameters and origins. However, one would like our theoretical understanding to be such that given a white dwarf binary of arbitrary masses and compositions at the time that the less massive component gets into contact, one could reconstruct the previous evolutionary pathways and the subsequent evolution to merger, tidal disruption or stable mass transfer.

Mass transfer in close DWD binaries has generally been taken to be stable if the mass ratio is smaller than 0.8. However, [6] identified the interesting possibility of the mass transfer stream that directly impact the surface of the accretor during this phase. The nature of it may depend both on the structure of the donor and the mass ratio of components. Thus, a stream of matter is pulled from the donor star through the inner Lagrangian point. If the matter stream does not impact the surface of the companion star, then the mass lost from the donor is expected to settle into a disc [7] and the division between the disc accretion and direct impact has been studied before ([8] [9] [10]). Torques exerted between the discs and the component white dwarfs allow angular momentum stored in the disc to be transferred back to the orbit ([7] [11]). However, the life time of these systems is defined not by feedback of angular momentum to orbit, but by angu- 
lar momentum loss from the systems due to GR. If direct impact drives the system apart, both [9] and [10] showed that a stabilizing accretion disk is likely to be created. If direct impact mass transfer increases the mass ratio and decreases the orbital period, the mass transfer rate may eventually become dynamically unstable, which can result in a merger.

As we have showed in [12] henceforth called paper I that mass transfer phase in binary stellar evolution can either decrease or increase the orbital period of the system, crucially depending on the exchange of angular momentum between the binary components, the orbit and the component spins. In order to calculate the angular momentum changes during mass transfer, both the studies of [9] and [10] utilize a numerical prescription based on [13] approximations to more accurately determine the flow of angular momentum between the component spins and the orbit. In that formulation, it is assumed that the angular momentum transferred from the orbit to the spin of the accretor is exactly equal to the angular momentum of the ballistic particle in a circular orbit around the donor at its average radius during its motion from donor to accretor ${ }^{1}$. The present study will building upon these studies and crucially depending on the changes in the orbital angular momentum, the period and radius of the donor stars and that of its Roche lobe in response to the mass transfer, providing direct ballistic integrations of the mass transfer stream using an approximation adopted from the [13]. This led us to extend the analytical and numerical solution of [14] for the time dependent behavior of the mass transfer by relaxing most of the assumptions made to provide the problem manipulable. Here, we retain the simplifying assumption of Roche lobe calculation, but allow all the binary parameters to vary self-consistently.

The aim of this paper is to determine the evolution of angular momentum and orbital period changes between the component spins and the orbit in close DWD binaries throughout GR, tides and mass transfer effects by providing different range of orbital parameters and stellar models in a full self-consistent manner. In particular, we examine the comparison between numerical and analytical solutions to determine these systems whether, and under what circumstances, dissipative tidal coupling of the accretor to the orbit, through direct impact, can stabilize the dynamical mass transfer. This paper is organized as follows:

In Section (2) we develop the basic differential equations which governing the evolution of orbital parameters, assuming mass transfer through some well-specified model(s) and the basic assumptions with dynamical stability of mass transfer and discuss the difference between this method and the method utilized by the previous studies. In Section (3) we find and discuss the numerical solutions of the systems. In Section (4) we illustrate the results and discussion of this paper in comparison to the previous studies. Finally our main conclusion is summarized in Section (5).

${ }^{1}$ [9] assume that the donor remains tidally locked, while [10] does allow for variation in the spin of the donor, but does not do so in a self-consistent manner. 


\section{Mass Transfer and Evolution of Orbital Parameters}

\subsection{Basic Assumptions}

In this paper, we consider a close binary system of two white dwarfs with masses $M_{d}$ and $M_{a}$, volume-equivalent radii of $R_{a}$ and $R_{d}$, and uniform rotational angular velocities of $\Omega_{a}$ and $\Omega_{d}$ with axes perpendicular to the orbital plane for the accretor and donor, respectively ${ }^{2}$. We assume that the mass of each star is distributed spherically symmetrically. The binary is assumed to be in an initially circular keplerian orbit with orbital period, $P_{o r b}$. The radius of each object is assigned following Eggleton's zero-temperature mass radius relationship of [13]. We chose the $P_{\text {orb }}$ of the orbit such that the volume-equivalent radius of the mass of the donor $\left(R_{d}\right)$ is equal to the volume-equivalent radius of its Roche lobe $\left(R_{L}\right)$ as fit by [15]. Both the $M_{d}$ and $M_{a}$ initially rotate synchronously with the orbit ${ }^{3}$.

\subsection{Method}

In this paper, we perform a Monte Carlo integration to calculate the volume-equivalent Roche lobe radius [16] over a two dimensional grid in $P_{o r b}$ and mass ratio, $q$ parameter space. We also apply Runge-kutta methods for numerically estimating solutions to basic differential evolution equations of obit, which will be discussed in Section (3). Hence, we determine the set of orbital parameters and their differential equations which governs the evolution of angular momentum and orbital period changes due to direct impact accretion in close DWD binaries, using the Eggleton function for the Roche lobe, $R_{L, E_{g g}}$ for calculating its size, the dynamical mass transfer rate, and the corresponding synchronization time scales for different cases, under certain restrictive approximations which determine the strength of tidal coupling. We now have all the steps in place to solve the evolution of these systems.

Our basic calculations are performed using a Monte Carlo integration method in a stationary inertial reference frame located at the initial center of mass of the binary system, which solving ordinary differential equations of orbital parameters at equivalent points. Following the above assumptions, we can investigate the outcomes for these systems with total mass, $M$ and the orbital parameters such as $P_{o r b}$ :

$$
P_{o r b}=\left(\frac{4 \pi^{2} a^{3}}{G M}\right)^{1 / 2},
$$

where $G$ and $a$ are the universal gravitational constant and semi-major axis, respectively, and taking the donor to be the less massive star, the one that fills its Roche lobe.

\subsection{Evolution of Angular Momentum}

As we have investigated in [12], as the matter is being transferred between the

${ }^{2}$ Throughout this paper, the subscripts "a" and " $\mathrm{d}$ " will correspond to the accretor and donor, respectively.

${ }^{3}$ If the donor rotates non-synchronously, the Roche lobe radii can be calculated as given in [16]. 
components in the binary systems, not only the mass ratio, $q$, but also the $P_{\text {orb }}$ will be changed due to angular momentum redistribution between the components of two stars. Then the total angular momentum, $J_{t o t}$, of the binary system is given by:

$$
J_{t o t}=J_{o r b}+\sum_{i=1}^{2}\left(J_{s p i n, i}\right) ; i \in(a, d)
$$

where $J_{o r b}$ is the orbital angular momentum, which is given by:

$$
J_{o r b}=M_{a} M_{d}\left(\frac{G a}{M}\right)^{1 / 2}
$$

and the spin angular momenta of the accretor and donor are $J_{\text {spin, } a}=K_{a} M_{a} R_{a}^{2} \Omega_{a}$ and $J_{\text {spin,d }}=K_{d} M_{d} R_{d}^{2} \omega_{d}$, respectively, where $K_{a}$ and $K_{d}$ are dimensionless constants depending upon the internal structure of the accretor and donor, respectively.

Here, we express the total angular momenta in terms of the rotational rates of the accretor, $f_{a}$ and donor, $f_{d}$ relative to the orbital angular velocities of the stars. Thus the rotational angular velocities of the stars, $\Omega_{i}$ can be written in terms of the rotational rates and the angular velocity of the circular orbit, $\Omega_{o r b}$ is given by

$$
\Omega_{i}=f_{i} \Omega_{\text {orb }} .
$$

Then we can rewrite the total angular momentum of the binary system as:

$$
J_{\text {tot }}=M_{a} M_{d} \sqrt{\frac{G a}{M}}+\sum_{i=1}^{2}\left(K_{i} M_{i} R_{i}^{2} f_{i}\right) \Omega_{o r b} .
$$

The form of the $J_{\text {orb }}$ term adopted above assumes the binary revolves at the Keplerian angular velocity of the circular orbit, $\Omega_{o r b}^{2}=G M / a^{3}$, which is a good approximation if the stars are centrally condensed.

Here, we introduce three effects that change the $J_{o r b}, P_{o r b}$ and $\Omega_{i}$ over time: mass transfer (MT), tides, and GR. Assuming each effect is independent of the others, we can then write the change of the orbital angular momentum as the sum of the change due to each of the above effects:

$$
\dot{J}_{o r b}=\dot{J}_{o r b, M T}+\dot{J}_{o r b, G R}+\sum_{i=1}^{2}\left(\dot{J}_{o r b, t i d e s, i}\right) \text {, }
$$

which is known as the orbital angular momentum balance equation. To determine the total change in the angular momentum, then, we simply need to write the change due to each of the above effects. A system of two point masses orbiting around each other, in circular orbits, radiates gravitationally ([17]). Then the change in orbital angular momentum due to GR for a circular orbit is given by:

$$
\dot{J}_{o r b, G R}=-\left(\frac{32}{5} \frac{G^{3}}{c^{5}} \frac{M_{a} M_{d} M}{a^{4}}\right) J_{o r b} .
$$

Prior to the onset of mass transfer, we assume that the spins and orbit to be synchronized, the binary orbit will shrink and the $P_{o r b}$ of the system will de- 
crease due to the effect of angular momentum via GR as seen in Equation (7). During this time, tidal coupling will act to circularize the binary as well as synchronize the spins of the stars with the orbit. As mass transfer begins, angular momentum will be exchanged between the spin of the component stars and the orbit. This will ultimately affect the dynamical stability of the mass transfer process. As in [10], we express the exchange of orbital angular momentum due to tides as:

$$
\left.\dot{J}_{\text {orb tides }}=\sum_{i=1}^{2}\left(\frac{K_{i} M_{i} R_{i}^{2}}{\tau_{i}}\left(f_{i}-1\right) \Omega_{\text {orb }}\right)\right) .
$$

The term in the bracket of Equation (8) represent the torque due to dissipative coupling upon the accretor and donor. Hence, torques are parameterized in terms of the synchronization time scales ([18]) of the accretor, $\tau_{a}$, donor, $\tau_{d}$ and are linearly proportional to the difference between the component and the orbit spin, which determines the strength of tidal coupling relative to $M T$ and GR. We assume that the tidal synchronization time scales change because the masses and stellar radii relative to the orbital period are changing. As [10], we explore two different values for the synchronization time scale at contact: $\tau=10^{15}$ years (very weak tidal coupling) and $\tau=10$ years (very strong tidal coupling), which may be a better approximation for systems with short orbital periods, as we consider in our analysis.

In this work, we are only interested in direct impact mass transfer and orbital changes due to ballistic integrations where the particle impacts the surface of the accretor within one orbital period. In this case, the evolution of orbital parameters is determined by the differential equations, which will be given in Section (2.4). If the particle accretes within one orbital period, we classify this as a direct impact. If the particle does not accrete within one orbital period, it is likely that the ejection stream from the donor will self-intersect, resulting in the eventual formation of an accretion disk.

To determine $\dot{J}_{o r b, M T}$, we use the ballistic mass transfer calculations of [19] to examine the instantaneous effect of mass transfer in close DWD binary systems. This method uses a fully self-consistent, conservative, ballistic model of the transferred mass to determine the orbital parameters of the system after a single mass transfer event. Then, the change in the orbital parameters per unit mass that results is directly proportional to the mass transfer rate of our evolving DWD, $\dot{M}_{d}$ :

$$
\dot{J}_{o r b, M T}=\left(\frac{\Delta U_{o r b, b}}{M_{P}}\right) \dot{M}_{d},
$$

where $\Delta J_{o r b, b}$ is the change in the orbital angular momentum for a close DWD binaries as calculated by the above ballistic model for a single mass transfer event ejecting a particle of mass $M_{P}$. In this method, changes in $J_{o r b, M T}$ are calculated at each time step by integrating the three-body system consisting of the two stars and the discrete particle representing the mass transfer stream. The 
change in the $J_{\text {orb,MT }}$ per unit mass transferred is independent of the mass of the ejected particle as long as $M_{P} \ll M_{d}, M_{a}$.

\subsection{Differential Equations for the Evolution of Orbital Parameters}

As we investigated the rate of change of orbital angular momentum in Section (2.3), we develop the equations for the evolution of orbital period and the rotational angular velocities of the stars. It follows from Equation (3) and [12] that

$$
\frac{\dot{J}_{o r b}}{J_{o r b}}=(1-q)\left(\frac{\dot{M}_{d}}{M_{d}}\right)+\frac{1}{3} \frac{\dot{P}_{o r b}}{P_{o r b}}
$$

for conservative mass transfer $(\dot{M}=0)^{4}$ and $q=\frac{M_{d}}{M_{a}}$. Since $\dot{M}_{a}=-\dot{M}_{d}$.

Hence for the conditions $M=$ constant and $J_{o r b}=$ constant it then follows that:

$$
\frac{\dot{P}_{o r b}}{P_{o r b}}=-3\left(\frac{\dot{M}_{a}}{M_{d}}\right)(q-1)>0 \text {. }
$$

\subsubsection{Evolution of the Orbital Period}

We examine the changes of the $P_{o r b}$ due to dynamical mass transfer, tides and GR. We assume that each effect is independent, and write the total change in the $P_{o r b}$ due to each of the above effects, respectively, as:

$$
\dot{P}_{o r b}=\dot{P}_{o r b, M T}+\dot{P}_{o r b, G R}+\sum_{i=1}^{2}\left(\dot{P}_{o r b, t i d e s, i}\right) .
$$

To calculate $\dot{P}_{o r b, M T}$, we utilize the model for mass transfer developed in [19]. Using this models, we calculate the time rate of change of $P_{\text {orb }}$ due to mass transfer rate as:

$$
\dot{P}_{o r b, M T}=\left(\frac{\Delta P_{o r b, b}}{M_{P}}\right) \dot{M}_{d} .
$$

Here, $\Delta P_{o r b, b}$ is the change in the orbital period for a close DWD binaries as calculated by the above ballistic model of [19] as described in Section (2.3) for the evolution of orbital angualar momentum.

Next we calculate $\dot{P}_{o r b, t i d e s}$ and $\dot{P}_{o r b, G R}$. As noted by [9] and [10], we used a different metric for changing the spin angular momentum due to tides of Equation (8). Hence we develop $\dot{P}_{\text {orb,tides }}$ from that angular momentum change. Holding the donor mass constant $(\dot{M}=0)$ for the final two terms of Equation (12) we can combine Equation (6) with Equation (11) to obtain:

$$
\dot{J}_{\text {orb,tides }}+\dot{J}_{o r b, G R}=\left(\frac{1}{3 P_{o r b}}\left(\dot{P}_{o r b}\right)_{\text {tides }, G R}\right) J_{o r b},
$$

where $\left(\dot{P}_{\text {orb }}\right)_{\text {tides }, G R}$ is the total change of the orbital period due to the combined effects of tides and GR. Assuming as before that changes to the orbital period due to tides and changes due to GR are independent we can rewrite the above as: ${ }^{4}$ Recall that we assume the orbit remains circular in this paper. 


$$
\dot{J}_{o r b, t i d e s}+\dot{J}_{o r b, G R}=\left(\frac{\dot{P}_{o r b, t i d e s}}{3 P_{o r b}}+\frac{\dot{P}_{o r b, G R}}{3 P_{o r b}}\right) J_{o r b} .
$$

Using Equation (3), Equation (7), and Equation (8), it follows that:

$$
\dot{P}_{\text {orb }, \text { tides }}=\frac{3 P_{\text {orb }}}{\mu \sqrt{G a M}} \sum_{i=1}^{2}\left(\frac{K_{i} M_{i} R_{i}^{2}}{\tau_{i}} \omega_{i}\right) \text {, }
$$

and

$$
\dot{P}_{o r b, G R}=-\frac{96}{5} \frac{G^{3}}{c^{5}} \frac{M_{a} M_{d} M}{a^{3}} .
$$

From Equation (4) and Equation (8) we can re-write Equation (16) as

$$
\dot{P}_{\text {orb }, \text { tides }}=\frac{3}{\mu\left(P_{o r b} M^{2}\right)^{1 / 3}}\left(A_{a}+D_{d}\right),
$$

where

$$
A_{a}=\frac{K_{a} M_{a} R_{a}^{2}}{\tau_{a}}\left(\frac{\Omega_{a}-\Omega_{o r b}}{\Omega_{o r b}}\right),
$$

and

$$
D_{d}=\frac{K_{d} M_{d} R_{d}^{2}}{\tau_{d}}\left(\frac{\Omega_{d}-\Omega_{o r b}}{\Omega_{o r b}}\right) .
$$

Finally, inserting Equation (13), Equation (17), \& Equation (18) into Equation (12) leads to the equation for the dynamical evolution of the orbital period with time as:

$$
\dot{P}_{o r b}=\left(\frac{\Delta P_{o r b, b}}{M_{P}}\right) \dot{M}_{d}+\frac{3\left(A_{a}+D_{d}\right)}{\mu\left(P_{o r b} M^{2}\right)^{1 / 3}}-\dot{P}_{o r b, G R} .
$$

\subsubsection{Evolution of the Rotational Angular Velocities of the Stars}

We derive the equations for the evolution of the components spins, $\Omega_{a}$ and $\Omega_{d}$. Thus the changes in both components can be defined as:

$$
\dot{\Omega}_{i}=\dot{\Omega}_{i, M T}+\dot{\Omega}_{i, \text { tides }}+\dot{\Omega}_{i, G R} .
$$

Analogous to $\dot{P}_{M T}$ in Equation (13), the change in $\Omega_{i}$ due to mass transfer, $\dot{\Omega}_{i, M T}$ can be expressed as:

$$
\dot{\Omega}_{i, M T}=\left(\frac{\Delta \Omega_{b, i}}{M_{P}}\right) \dot{M}_{d}
$$

Here, $\Delta \Omega_{b, i}$ is the change in the rotational angular velocities of the star $i$ resulting from a single mass transfer as described in Section (2.3). As in Equation (4), the spin angular momentum of each component can be written as:

$$
J_{\text {spin }, i}=K_{i} M_{i} R_{i}^{2} \Omega_{i},
$$

where $\Omega_{i}=f_{i} \Omega_{\text {orb }}$. Hence, we consider that the tidal forces exist to redistribute angular momentum, working to keep the spins of the donor and accretor syn- 
chronous with the orbit $\left(f_{i}=1\right)$. Now we can determine $\dot{\Omega}_{i, \text { tides }}$ and $\dot{\Omega}_{i, G R}$ by differentiating Equation (22) with respect to time assuming the mass held constant:

$$
\begin{gathered}
\dot{J}_{\text {spin }, i}=K_{i} M_{i} R_{i}^{2}\left(\dot{\Omega}_{i, \text { tides }}+\dot{\Omega}_{i, G R}\right) \\
-\frac{2}{3} K_{i} M_{i} R_{i}^{2} \frac{\Omega_{i}}{P_{o r b}}\left(\dot{P}_{\text {tides }}+\dot{P}_{G R}\right) .
\end{gathered}
$$

The second term in Equation (24) depends only upon changes due to tides and GR. Since we do not include any GR effect on the spin angualar momentum of the components, conservation of angular momentum indicates that any changes in the spin angualar momentum of a component must be equal and opposite to the changes in the orbital angular momentum of the system due to tides acting on that component. Using Equation (4) and Equation (8), we have:

$$
\dot{J}_{\text {spin, } i}=-\sum_{i=1}^{2} \frac{K_{i} M_{i} R_{i}^{2}}{\tau_{i}}\left(\Omega_{i}-\Omega_{\text {orb }}\right) .
$$

By combining Equation (24) and Equation (25) we can derive the expression for $\dot{\Omega}_{i, \text { tides }}+\dot{\Omega}_{i, G R}$ as:

$$
\dot{\Omega}_{i, \text { tides }}+\dot{\Omega}_{i, G R}=-\frac{\Omega_{i}}{\tau_{i}} \frac{2}{3} \frac{\Omega_{i}}{P_{o r b}}\left[-\beta_{G R}+\frac{3\left(A_{a}+D_{d}\right)}{\mu\left(P_{o r b} M^{2}\right)^{1 / 3}}\right],
$$

where

$$
\beta_{G R}=\frac{96 G^{3} M_{a} M_{d} M}{5 c^{5} a^{3}} .
$$

Using Equation (21), Equation (22) and Equation (26) we obtain the equations for the evolution of $\Omega_{a}$ and $\Omega_{d}$ as:

$$
\begin{gathered}
\dot{\Omega}_{a}=\left(\frac{\Delta \Omega_{b, a}}{M_{P}}\right) \dot{M}_{d}-\frac{\Omega_{a}}{\tau_{a}}+\frac{2}{3} \frac{\Omega_{a}}{P_{o r b}} \\
{\left[-\beta_{G R}+\frac{3\left(A_{a}+D_{d}\right)}{\mu\left(P_{o r b} M^{2}\right)^{1 / 3}}\right],}
\end{gathered}
$$

and

$$
\begin{gathered}
\dot{\Omega}_{d}=\left(\frac{\Delta \Omega_{b, d}}{M_{P}}\right) \dot{M}_{d}-\frac{\Omega_{d}}{\tau_{d}}+\frac{2}{3} \frac{\Omega_{d}}{P_{o r b}} \\
{\left[-\beta_{G R}+\frac{3\left(A_{a}+D_{d}\right)}{\mu\left(P_{o r b} M^{2}\right)^{1 / 3}}\right],}
\end{gathered}
$$

\subsection{Mass Transfer Rate}

The nature of mass transfer and its stability is very important in a close DWD binaries. It is rooted in a similar treatment of mass transfer under consequential angular momentum losses via GR [20]. Whether, once started, mass transfer will 
proceed in a stable or dynamically unstable manner depending on the response of the mass losing donor star and its Roche lobe to the mass transfer. For all types of donor star, the mass transfer rate is a strong function of the depth of contact, defined here as the amount by which the donor overflows its Roche lobe as:

$$
\Delta=R_{d}-R_{L},
$$

where $R_{d}$ is the radius of the donor and $R_{L}$ is the radius of its Roche lobe. The mass loss rate from the donor monotonically increases with $\Delta$. The way in which the mass transfer rate varies with $\Delta$ has been investigated in many analyses (see, for example, [21] [22] [23]). The correct way to treat this is to compute the stellar structure ([23]). In accordance with [9], we approximate the mass transfer as adiabatic ([24]). In the adiabatic response, which for a white $\mathrm{d}$ warf donor gives a mass transfer rate of

$$
\dot{M}_{d}=-f\left(M_{a}, M_{d}, a, R_{d}\right) \Delta^{3},
$$

for $\Delta>0$, and zero for $\Delta<0$. Combining results from [24] [25] and [22], the function $f$ is given by:

$$
f\left(M_{a}, M_{d}, a, R_{d}\right)=\frac{8 \pi^{3}}{9}\left(\frac{5 G m_{e}}{h^{2}}\right)^{3 / 2} \frac{\left(\mu_{e}^{\prime} m_{n}\right)^{5 / 2}}{P_{o r b}} \Lambda,
$$

where $\Lambda=\left(3 \mu M_{d} / 5 r_{L} R_{d}\right)^{3 / 2}\left[a_{d}\left(a_{d}-1\right)\right]^{-1 / 2}, m_{e}$ is the mass of an electron, $m_{n}$ is the mass of a nucleon, $\mu_{e}^{\prime}$ is the mean number of nucleons per free electron in the outer layers of the donor (which we will assume to be two) and $\mu$ and $a_{d}$ are parameters associated with the Roche potential

$$
\mu=\frac{M_{d}}{M_{a}+M_{d}} \text { and } a_{d}=\frac{\mu}{X_{L 1}^{3}}+\frac{1-\mu}{\left(1-X_{L 1}\right)^{3}} .
$$

where, $X_{L 1}$ is the distance from the center of the donor to the inner Lagrangian point of the donor, in units of the semi-major axis, a.

\subsection{Roche Model}

In the case of [9], where the rotational angular velocity of the donor is fixed to the orbital velocity and the orbit is circular throughout, the shape and volume of the Roche lobe depends only upon the mass ratio and the semi-major axis of the system. For higher mass ratio, stars become more and more deformed. Thus mass transfer is dynamically unstable. In that case, we introduce the effective radius, $r_{L}$, of the Roche lobe, the most widely used approximation being from [15],

$$
r_{L}=\frac{0.49 q^{2 / 3}}{0.69 q^{2 / 3}+\log \left(1+q^{1 / 3}\right)},
$$

effectively, it is a tidal radius where mean density in lobes are equal:

$$
R_{L}=r_{L} a,
$$

for $0.1 \leq q \leq 1$ and so with the notation of [9], we have 


$$
\frac{\dot{R}_{L}}{R_{L}}=\zeta_{r_{L}} \frac{\dot{M}_{d}}{M_{d}}+\frac{2}{3} \frac{\dot{P}_{o r b}}{P_{o r b}}
$$

where $\zeta_{r_{L}}$ takes values between 0.33 and 0.48 is the logarithmic derivative of $r_{L}$ with respect to $M_{d}$ for both [26] and [15] small $q$ approximation for the Roche lobe radius. In the same spirit we write the logarithmic time derivative of the donor radius, $R_{d} \equiv R_{d}\left(M_{d}, t\right)$ as:

$$
\frac{\dot{R}_{d}}{R_{d}}=D+\zeta_{a d} \frac{\dot{M}_{d}}{M_{d}}
$$

where $D=\left(\partial \log \left(R_{d}\right) / \partial \log (\partial t)\right)_{M_{d}}$, represents the rate of change of the donor radius due to intrinsic processes such as thermal relaxation and nuclear evolution, whereas $\zeta_{a d}$ usually describes changes resulting from adiabatic variations of $M_{d}$ [27]. If the donors are degenerate as in the white dwarf case, $\zeta_{a d}$ is simply obtained from the equilibrium mass radius relationship for the donor ${ }^{5}$. As $\zeta_{a d}$ and $\zeta_{r_{L}}$ are both monotonic functions of mass loss, it is a sufficient condition that mass transfer be stable at the onset of Roche lobe overflow in order to ensure dynamical stability of mass transfer, which depends on the tidal coupling between the accretor and the orbit undergoing direct impact accretion. The larger $\zeta_{a d}$, the more stable the mass transfer is. To consider stability in detail, we will use the linear stability analysis following ([11] [28]).

\section{Numerical Solutions}

We integrate numerically the orbital evolution Equation (2), Equation (4), Equation (6), Equation (11), Equation (20), Equation (28), Equation (29), Equation (31), Equations (35)-(37) using $6^{\text {th }}$ and $8^{\text {th }}$ order Runge-Kutta ordinary differential equation solver [29], allowing the binary parameters to adjust self-consistently. We consider that the orbital angular momentum of the system are conserved throughout the integration over the entire parameter space. Specifically, we compute the angular momentum and orbital period changes between the component spins and orbit in close DWD binaries undergoing mass transfer through direct impact of the transfer stream, allow the binary separation to change and compute the values of $\zeta_{a d}$ as it evolve. Here, we use Eggleton's approximation of the zero-temperature mass radius relationship cited by [13] and [9], which is a good approximation for systems containing white dwarfs. Also, a $\zeta_{a d}$ significantly different from -0.33 clearly affects the stability and evolution of the systems at the onset of mass transfer, and can lead to shrinking orbits even if the mass transfer is stable with $\dot{M}_{d}=0$. In our subsequent analysis, where we are concerned about the long term integrations of the orbital evolution equations, we set $\left(\partial \log R_{d} / \partial t\right)_{M_{d}}=0$ and use the zero-temperature mass-radius relationship.

${ }^{5}$ The radius of each object is assigned following Eggleton's zero temperature mass radius relation ([13]). We assume that both the donor and accretor initially rotate synchronously with the orbit. 
In our integrations, we need to either assume or determine from other assumptions how the mass and angular momentum are redistributed in a close DWD binary system during mass transfer. This depends on the mode of accretion appropriate for the binary considered: does the stream impact the accretor or is an accretion disk present; is the mass transfer sub-Eddington and conservative, or are mass and angular momentum being ejected from the system following super-Eddington mass transfer. For most of the numerical integrations that follow, we use the appropriate rate of change of orbital angular momentum due to GR, MT and tides. However, if one is interested in the relatively rapid phases of dynamical mass transfer that follow contact and onset, then the qualitative properties of our integrated evolutions depend strongly on these assumptions.

Figure 1 shows the evolution of the orbital period and angular momentum conservation with their spin angular momentum of the accretor and donor in close DWD binaries over the entire parameter space of interest for integrations that calculated using the Eggleton approximation for the Roche model. In this result, we are only interested in direct impact mass transfer where the particle impacts the surface of the accretor within the orbital periods. Compared to direct impact accretion, disc accretion is provide a basic mechanism for redistributing spin angular momentum in the accretor back into the orbit through tidal coupling in [7]. As a result, it is likely that once a system enters a phase of disc accretion it will remain in this phase or perhaps even become detached as the orbital period continues to grow and the mass ratio decreases due to continued mass transfer. Thus if a disk is formed at any point the integration stops and the system is assumed to be stable throughout its lifetime.

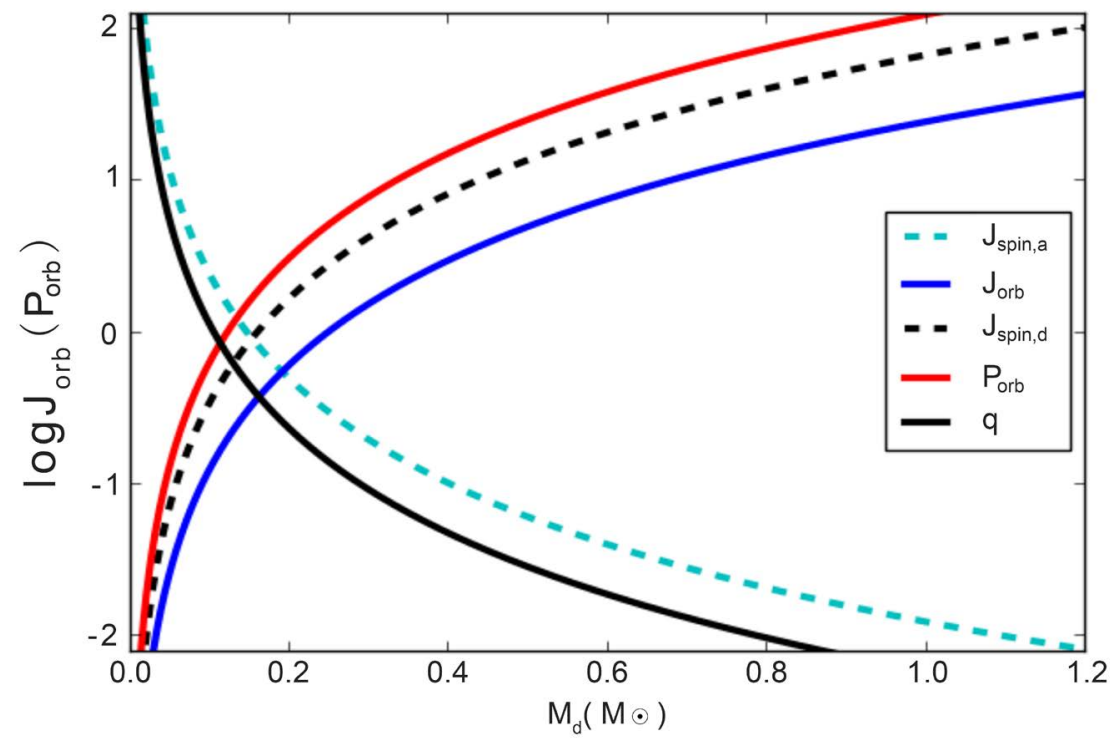

Figure 1. The evolution of orbital period and angular momentum conservation with their $J_{\text {spin,a }}$ and $J_{\text {spin,d }}$ over entire close DWD binary system, which obtained using the Eggleton approximation from Equation (5) for the calculation of the Roche lobe overflow and rapid mass transfer stream. 
As a system evolves, it is possible to pass back and forth through phases of mass transfer and phases of no mass transfer as the orbital period and the two masses change. If $P_{\text {orb }}$ increases enough for mass transfer to stop altogether, the integration proceeds (with $\dot{M}_{d}=0$ ) until the action of GR shrinks the orbit sufficiently for mass transfer to resume rather than the analytical one. We integrate over a period of $1.25 G \mathrm{yr}$. As in [9], if $\dot{M}_{d}$ less than $0.1 M_{\odot} \mathrm{yr}^{-1}$ at any point during the integration, the integration continued and the system is desired as dynamically stable.

In Figure 2 we show the approximate adiabatic mass radius exponent, $\zeta_{a d}$ from Equation (37) using the Eggleton's approximation of the zero-temperature mass radius relationship [13] for stars with initial masses from $0.12 M_{\odot}-0.78 M_{\odot}$ and the corresponding mass radius relations. These were calculated by assuming a constant mass loss rate of $10^{-5} M_{\odot} \mathrm{yr}^{-1}$. The large initial values for $\zeta_{a d}$ imply that the star has to lose very little mass to shrink significantly. This simply represents the fact that, in these systems, a large fraction of the envelope contains very little mass.

\subsection{Angular Momentum and Orbital Period Changes Due to Direct Impact Accretion}

In this section, we investigate the numerical integrations for the change in the evolution of orbital angular momentum per unit transferred mass, which is independent of the mass of the ejected particle as long as $M_{P} \ll M_{d}$ from Equation (9) following a single ballistic orbit as a function of the donor mass for white dwarfs undergoing direct impact mass transfer. Figure 3 shows the evolution of orbital angular momentum change per unit transferred mass, $\dot{J}_{\text {orb,MT }}$ and period changes from Equation (9) and Equation (11), respectively, with the less massive donor and the massive accretor stars. Hence, we compared the results of

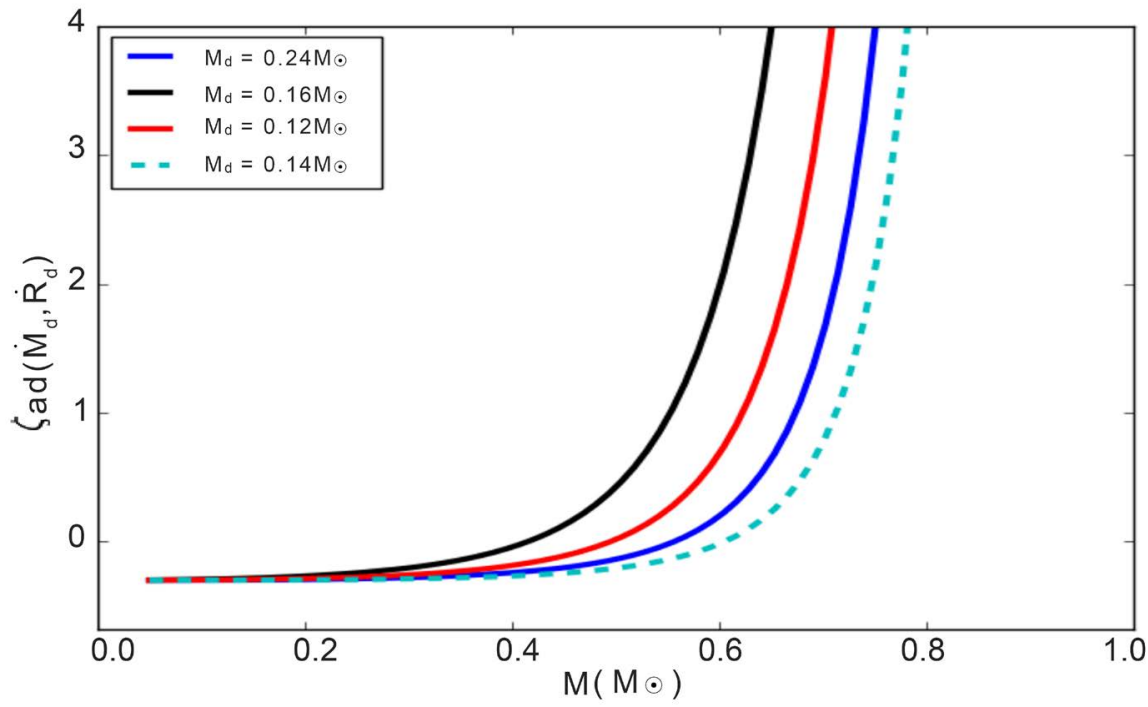

Figure 2. Results of the approximate adiabatic mass radius exponents over the total mass of the system for evolved stars of initial masses $0.12 M_{\odot}, 0.14 M_{\odot}, 0.16 M_{\odot}, 0.24 M_{\odot}$. 


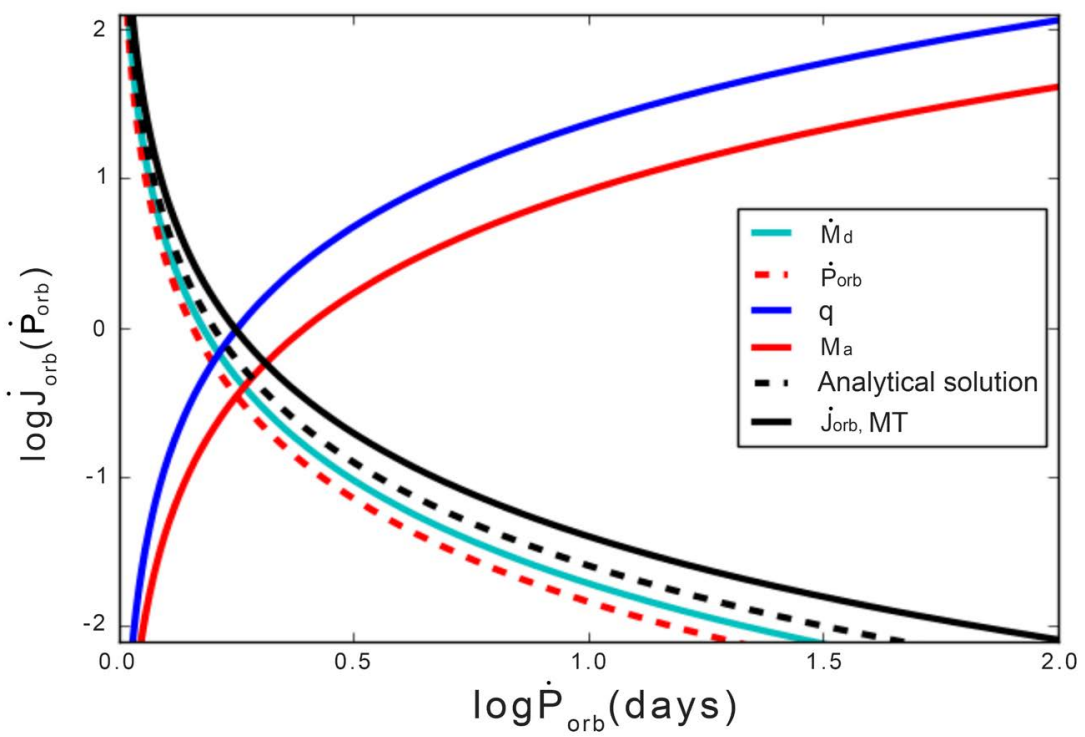

Figure 3. The $\dot{J}_{o r b, M T}$ per unit transferred mass correspond to variations in the mass of accretor and mass ratios of the systems versus $\dot{P}_{o r b}$. Here, in our calculation the region above the $\dot{J}_{o r b, M T}$ shows the unstable mass transfer whereas the region below the $\dot{M}_{d}$ shows the stable mass transfer. Hence, the dashed black line represents the analytical solution between these stable and unstable systems.

the $\dot{J}_{o r b, M T}$ and $\dot{M}_{d}$ due to direct impact accretion with the $\dot{P}_{o r b}$ and $M_{a}$. As expected, our numerical results agree quite well to this analytic solution.

In Figure 4 we investigate a single ballistic integration as a function of the masses of the donor and accretor. The solid blue and dashed black lines above a solid cyan line are a region corresponds to systems that undergo direct impact and reach stable configuration, while below this solid cyan line such as solid black and dashed red lines are a region corresponds to systems that undergo deformation. The solid cyan line between these two regions shows the analytical solution to this region, which was first derived and presented by [6] [9]. Thus, the change in the orbital angular momentum appears to become more positive with increasing donor mass. Following them, we calculate this line by taking the distance of closest approach for a ballistic integration given by [30]; as analytically fit by [6] and setting it equal to the radius of the accretor (as given by Eggleton's zero temperature mass radius relationship of [13]. In calculating this line, we assume the donor (also described by the same zero temperature mass radius relationship) completely fills its Roche lobe ([15]).

Following the above consideration, we show the orbital angular momentum changes per unit transferred mass in the parameter space of close DWD binaries with a low donor mass are more likely to remain stable over long periods of time, while the majority of the parameter space is expected to be unstable. Thus, we further investigate these parameter space from deformation to direct impact where the orbital angular momentum decreases more rapidly than previous studies, which shown as in solid red line. 


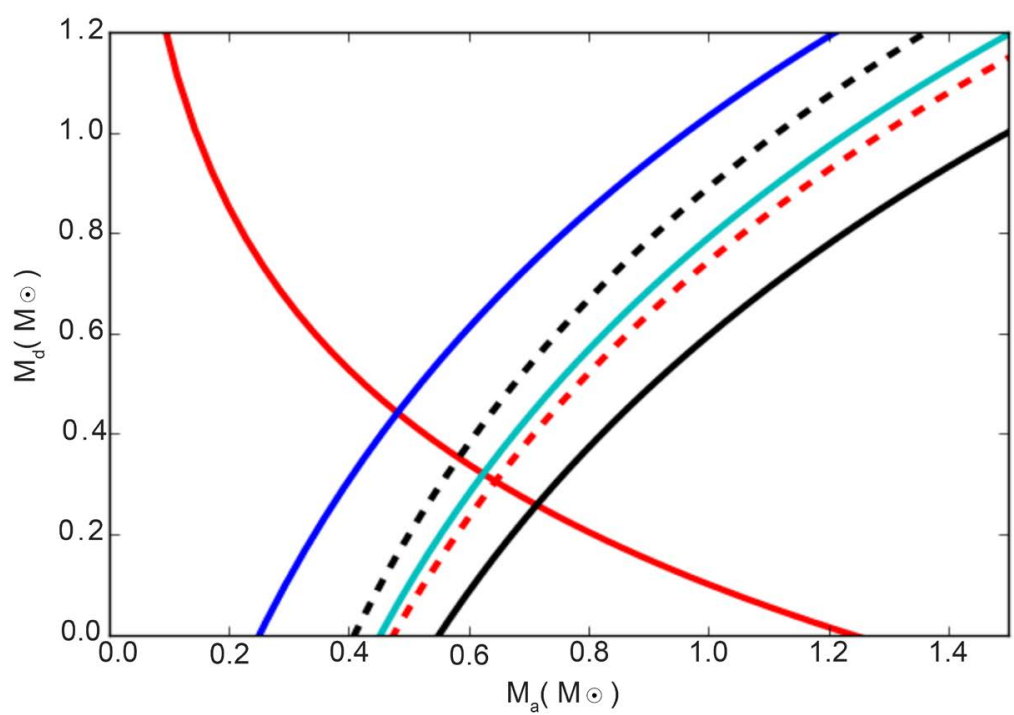

Figure 4. Result of a single ballistic integration as a function of the masses of the donor and accretor, which undergoing direct impact accretion from Equation (9).

\subsection{Evolution of Systems with Strong Tidal Coupling}

It is commonly assumed ([9]; but see also [10]) that the rotational angular velocity of the donor will remain synchronized with the angular velocity of the circular orbit. This is generally expected since Roche lobe overflow requires extreme tidal distortions in the donor star which act to synchronize its rotational angular velocity on short time scales. The accretor is generally not affected as strongly by tidal synchronization since, being more massive, it is not expected to be significantly tidally distorted. Some tidal coupling between the donor and the orbit is generally assumed, though it is not expected to be strong enough to keep the accretor from becoming a rapid rotator.

In Figure 5 we present the orbital parameters of the tides with initial tidal synchronization time scale of $\tau=10$ years : 1) the evolution of the rate of change of the spin angular momenta per unit transferred mass of the the donor ( $\dot{J}_{\text {spin, } d}$, for $\Omega_{d}=0.25$ ) and accretor $\left(\dot{J}_{\text {spin }, a}\right.$, for $\Omega_{a}=0.8$ ) from Equation (25), 2 ) the evolution of the rate of change of orbital angular momentum and period corresponds to the mass transfer rate from Equation (10) and Equation (31), respectively, and 3) the effective Roche lobe radius of the donor stars, which shows the end evolution of Roche lobe overflow. In general, systems with a smaller mass conserve orbital angular momentum better than systems with a higher mass. Systems where the orbital angular momentum conservation is larger tend to be systems where the numerical integration runs for many orbits, allowing systematic errors in the numerical integration to accumulate. Even so, all systems presented in this paper conserve mass and orbital angular momentum to better than $1 \%$ throughout the entire evolution.

\section{Results and Discussion}

As we developed and discussed the basic equations for the evolution of orbital 


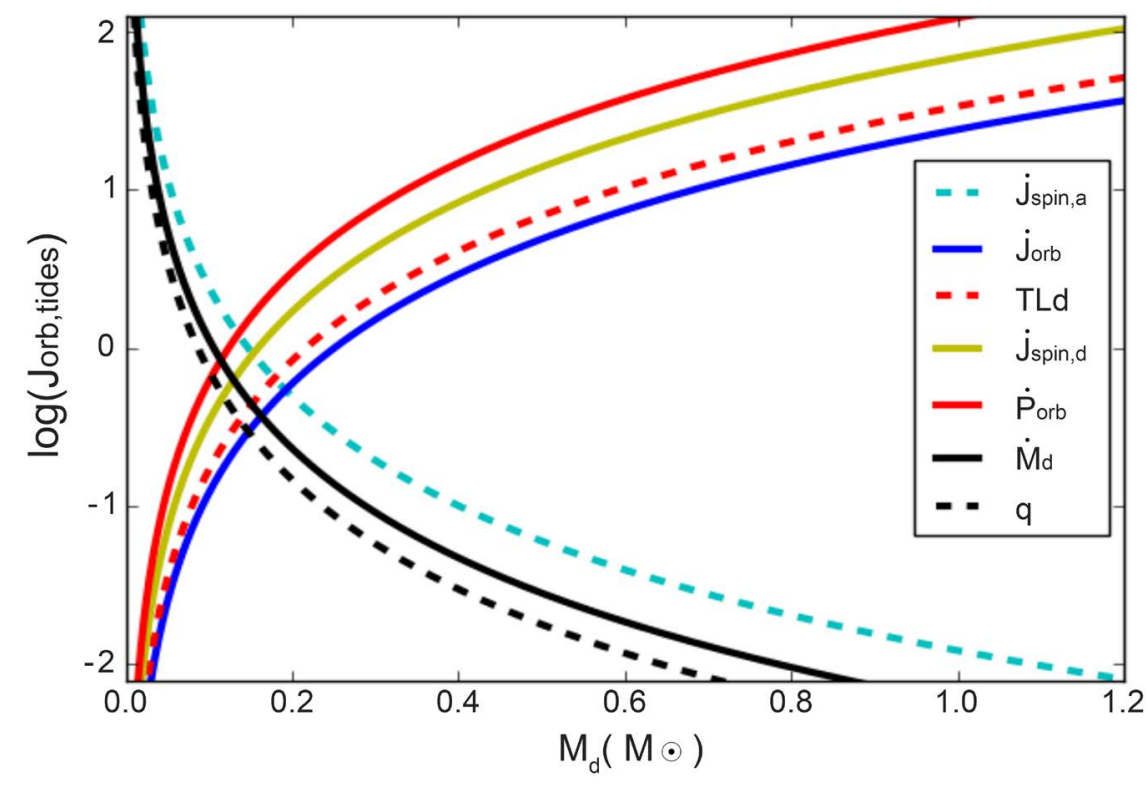

Figure 5. Results of evolution for the orbital parameters of the tides generating body with initial tidal synchronization time scale of $\tau=10$ years using the Eggleton approximation for the Roche lobe that can be calculated from Equation (8), Equation (10), Equation (25) and Equation (31). It shows the rate of change of orbital angular momentum between the donor and the orbit, i.e., the $\dot{J}_{\text {spin,d }}$, and $\dot{J}_{\text {spin, },}$ correspond to the rotational angular velocities of the donor and accretor, respectively. Here, the rotational angular velocity of the donor's, $\Omega_{d}=0.86$ remains synchronized to the rotational angular velocity of the circular orbit, $\Omega_{\text {orb }}=1.25$. Hence, we assume the angular momentum that would be removed from the spin of the donor is immediately returned from the orbit.

parameters in Sections (2) and (3), respectively, we compute the evolution over a grid in $M_{d}, M_{a}$ and $P_{o r b}$ parameter space for two different tidal synchronization time scales at contact: $\tau=10^{15}$ years and $\tau=10$ years to determine the dynamical stability of various systems. We also elaborate the comparisons between numerical and analytical solutions for the evolution of angular momentum and orbital period changes due to direct impact accretion in close DWD binaries by various stellar model approximations of mass transfer, with particular attention payed to the dynamical stability of these processes against runaway on synchronization time scales of the mass donating star.

\subsection{Evolution of Systems Using Eggleton Roche Lobe}

As we discussed in Section (3), Figure 5 shows the end result of systems with an initial synchronization time scale of $\tau=10$ years using $R_{L, E g g}$. Assuming that once a disc is reached, the system will remain stable for the remainder of its evolution, therefore we stop the integration once a disc is formed. However, with the exception of dynamically unstable systems, the evolution of all systems in this plot is stopped when the system reaches a phase of disc accretion. The time it takes to reach this phase of disc accretion varies from system to system.

As noted by [10] that allowing the spin of the donor to vary and by including 
the resulting effects of tidal coupling between the donor's spin and the orbit, the number of stable systems increased compared to the analysis of [9]. Hence, the main difference between our analysis and that of [10] is in the treatment of the way we manage the evolution of angular momentum and orbital period changes during the direct impact accretion process. In observing the increase in the number of stable systems compared to the analysis of [10], we conclude that by utilizing a mass transfer treatment that allows the rotation rates corresponds to rotational angular velocities of both components to vary and self-consistently accounts for the exchange of angular momentum between the spins of the components and the orbit, we are able to increase the number of stable systems.

Stronger tidal coupling will allow more spin angular momentum from the accretor to be transferred back into the orbit, which correspondingly slower increase in orbital period, causing the mass transfer rate and the rate of change of orbital angular momentum to decrease. This is to be expected and in agreement with the analyses of [10] and [9]. This results in an increase the stability of the systems in general.

In Figure 5 we have presented the dynamically stable mass transfer with increasing orbital period, but decreasing the mass ratio of the systems. However, in Figure 6 we have presented the dynamically unstable mass transfer with rapid increase in the orbital period for $\tau=10^{15}$ years is a direct result of the conservation of angular momentum: the angular momentum lost during the spin down and mass loss of the donor is greater than the angular momentum gained by the accretor. The net decrease in spin angular momentum corresponds to an increase in the orbital angular momentum, increasing the orbital period. The mass transfer rate increases rapidly as the mass loss from the donor causes the radius

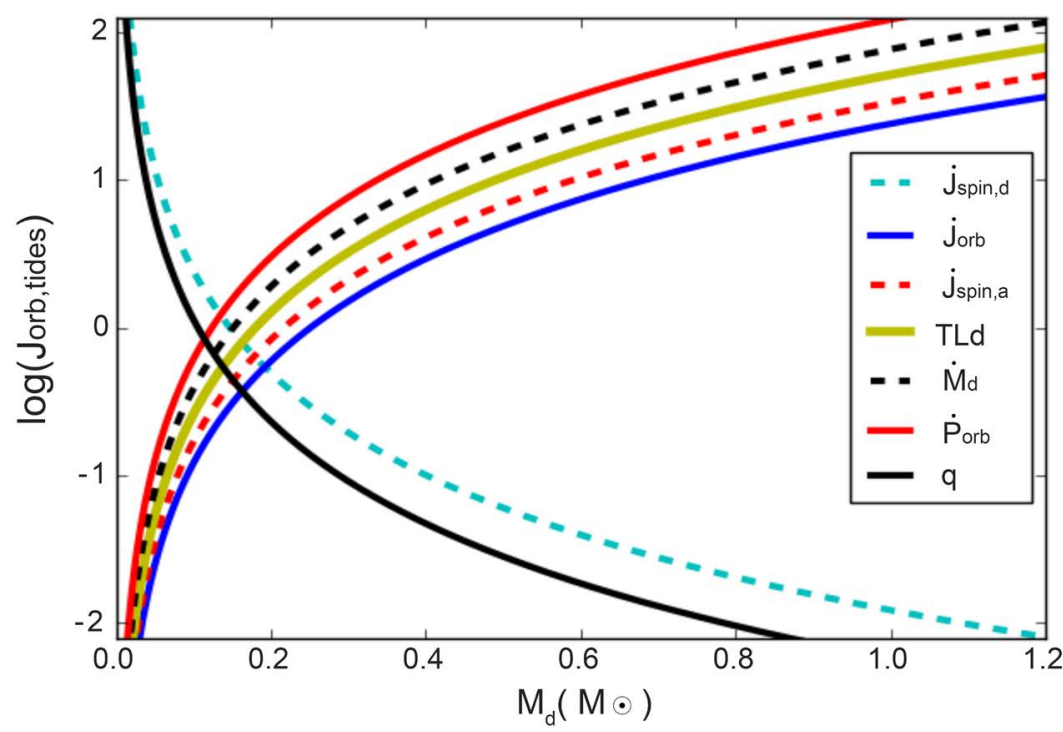

Figure 6. Same as in Figure 5, but with $\tau=10^{15}$ years using $R_{L, E g g}$ for the Roche lobe calculation. In this result, we observe that the $P_{o r b}$ increases due to the effect of tides. As a result $\dot{M}_{d}$ increases rapidly and the accretor spin increases significantly. 
to increase, even as the Roche lobe grows due to the increasing orbital period (see, e.g., Equation (30) and Equation (31)). Thus, we present the results of evolution for the orbital parameters of the tides generating body with tidal synchronization time scale of $\tau=10^{15}$ years. Here, we acknowledge the main limitation of our result to assume that systems are stable once they reach a phase of disc accretion. Systems with the less masses of the donor stars parameter space of interest here begin the integration in a disc phase, and are therefore labeled as stable. However, it is feasible that if these high donor mass systems were allowed to evolve through the initial disc phase, they would eventually become super-Eddington and potentially dynamically unstable.

\subsection{Comparison of System Evolution with analytical Solutions}

We have computed different orbital parameters of close DWD binaries and their evolution with synchronization time scales using $R_{L, E g g}$. Hence, for $\tau=10^{15}$ years, the system is dynamically unstable and for $\tau=10$ years the system is stable, but passes through a phase of super-Eddington accretion. In this section, we compare the analytical solutions of the various orbital parameters of these systems with the numerical solution that we investigated in Sections (3) and (4).

The rotational angular velocities of the component white dwarfs remain closer to synchronize. In our work, when we apply the $R_{L, E g g}$, the mass transfer rate will continue to increase until tides have transferred sufficient angular momentum from the spin of the components to the orbit to expanded the orbit and decrease the mass transfer rate. Thus, the mass transfer rate is decreased and tides continue to redistribute angular momentum between the component spins and the orbit (see, Figure 5). In Figure 7 we show the evolution of the mass radius relationship using the Eggleton approximation for the Roche lobe calculation. We also determine the evolution of the orbital period changes on a much longer time scale for the $R_{L, E g g}$ numerical solution than the analytical solution. This is due to the fact that, as mass transferred from the donor to the accretor, the mass ratio $q$ and the mass of the donor decreases relative to the orbit and the radius and mass of the accretor increases. These were calculated by assuming the constant mass loss rate of $1.25 \times 10^{-5} M_{\odot} \mathrm{yr}^{-1}$.

In Figure 8 we show the evolution of orbital parameters (i.e., the mass of the donor as a function of mass ratio) due to direct impact accretion. Hence, the solid blue line also indicates the deformed donor star at different moments in time. The solid green line show the evolution of orbital parameters for both synchronization time scales over which the mass transfer rate changes in Equation (31) for the non-deformed donor star whereas the dotted gray line is the deformed donor star and it is analytical solution, wich shows the evolution of the Roche lobe overflow. In general, the peak of solid gray, red, green, and blue lines indicate the evolution of the Roche lobe overflow in close DWD binaries through direct impact. 


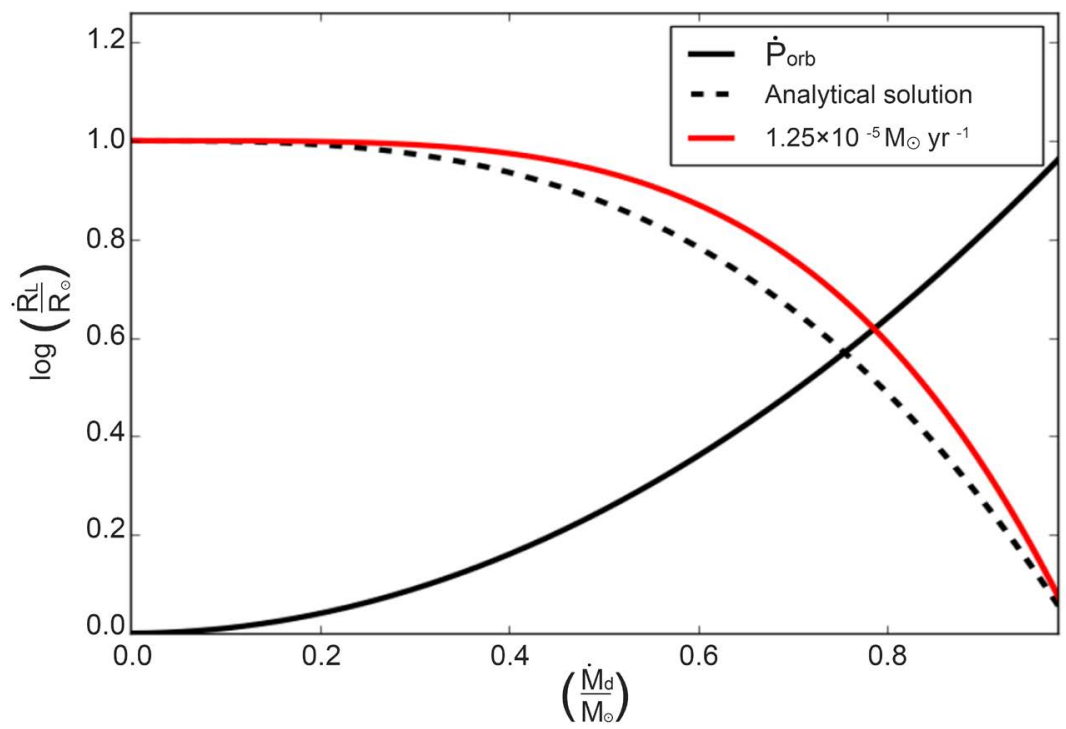

Figure 7. Result of the comparison between the numerical integrations and analytical solution for the evolution of the Roche lobe radius as a function of the mass of the donor stars for $\tau=10$ years with initial masses of $M_{d}=0.12 M_{\odot}$ and $M_{a}=0.8 M_{\odot}$ in a close DWD binary system. Hence $10^{3}$ years of evolution with solution obtained using $R_{L, E g g}$ for the calculation of the Roche lobe.

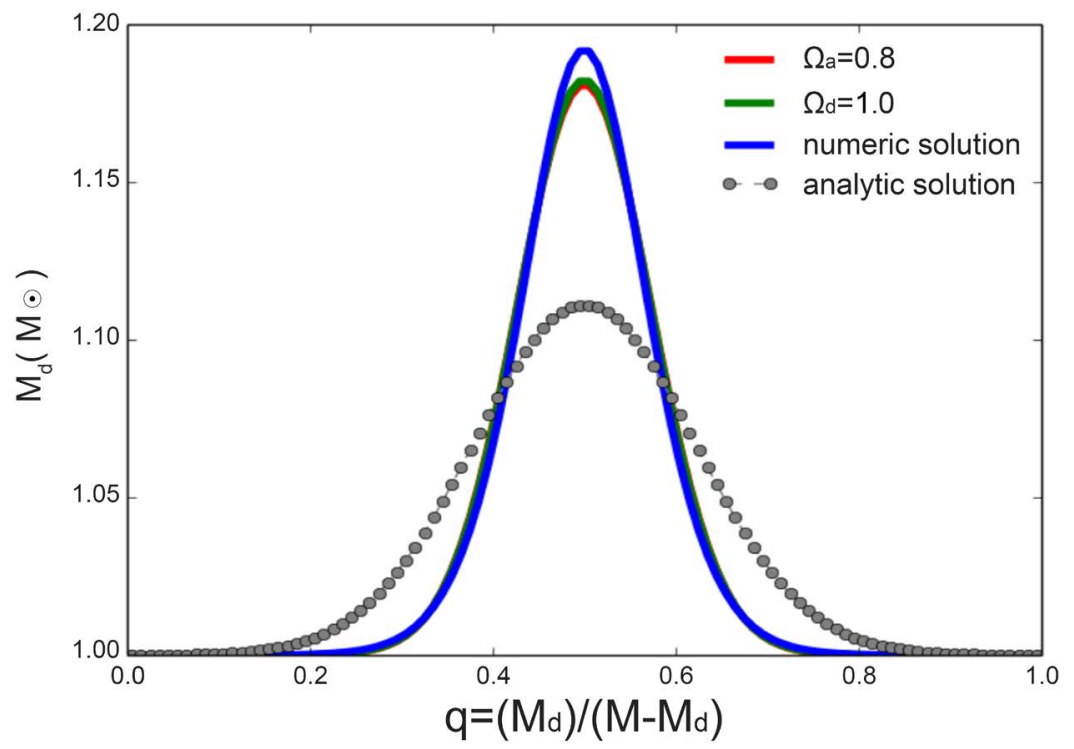

Figure 8. Results of the comparison between the numerical integrations and analytic solutions for the evolution of orbital parameters, i.e., $M_{d}\left(M_{\odot}\right)$ versus the mass ratio, $q$ with the rotational angular velocities of the donor, $\Omega_{d}$ and accretor, $\Omega_{a}$ on the Roche lobe radius in Equation (28) and Equation (29) for the second rotational angular velocity of the WD when the effect of the tides and rotation on the donors structure are ignored (solid red line) or included (green, blue and dotted gray lines).

Figure 9 shows the evolution of orbital parameters due to tidal coupling for $\tau=10$ years from Equation (28) and Equation (29). Thus, we numerically and 


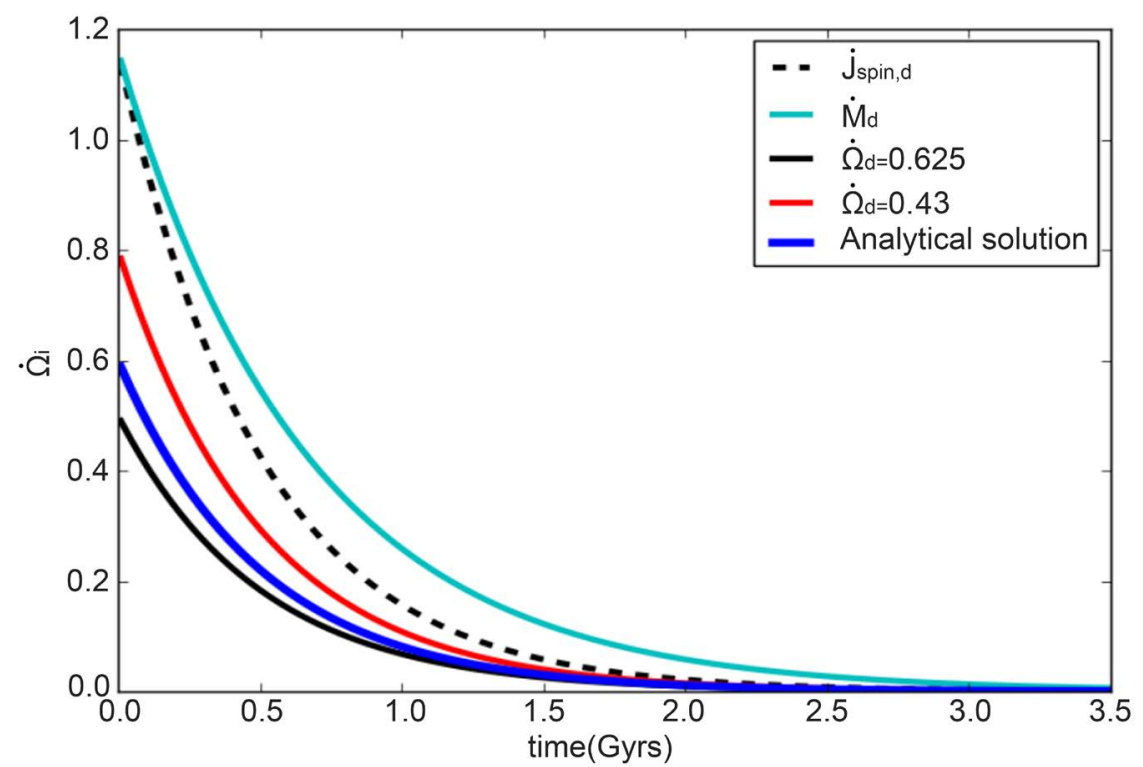

Figure 9. The comparison between the numerical and analytical solutions of orbital parameters for $\tau=10$ years. These results obtained using the $R_{L, E g g}$ calculation of the Roche lobe. In these systems the donor decreases its mass until it finally detached from the Roche lobe and forms a $0.28 M_{\odot}$ He WD and decrease the $\dot{J}_{\text {spin,d }}$.

analytically investigate the evolution of the rotational angular velocities of both donor and accretor stars in close DWD systems with initial masses of the donor, $M_{d}=0.86 M_{\odot}$ and accretor, $M_{a}=1.2 M_{\odot}$. In these systems, we provide a comparison between the numerical and analytical solution for the rate of change of the spin angular momentum for the accretor and the rate of change of the mass transfer rate corresponds to the $\dot{\Omega}_{d}$ and $\dot{\Omega}_{a}$, which shows our calculation stops when the donor reached $1.45 M_{\odot}$ (disc accretion).

\subsection{Comparison to Previous Results}

As it was studied by [8] [9] [10] that systems with a low donor mass are more likely to remain stable over long periods of time, while the majority of the parameter space (depending on the strength of the tidal coupling) is expected to be dynamically unstable. Here, by including the additional mass transfer effects presented here in a self-consistent way, DWD direct impact mass transfer may induce a stabilizing effect over a larger area of the parameter space.

As seen in Figures 1-5, we discussed the evolution of angular momentum and orbital changes due to direct impact and evolution of systems with strong tidal coupling. Also, in Figures 7-9, we investigated the comparison between the numerical and analytical solution of the systems for the evolution of parameters for $\tau=10$ years . However, as seen in Figure 6, the mass transfer rate increases initially, which causes the accretor to spin up and the donor to spin down. Hence, when we using $R_{L, E g g}$, the mass transfer rate will continue to increase until tides have transferred sufficient angular momentum from the spin of the components to the orbit to widen the orbit and decrease the mass transfer rate. Once this 
happens, the tides continue to redistribute angular momentum between the component spins and the orbit (see, Figure 5). When the system is dynamically unstable, the time scale for changes in the mass transfer rate becomes greater than the time scale at which the orbital period increases due to the effect of tides. As a result, the mass transfer rate increases rapidly and the spin of the accretor increases significantly.

\section{Conclusions}

We have presented the evolution of angular momentum and orbital period changes between the component spins and the orbit in close DWD binaries undergoing mass transfer through direct impact accretion, including the effects due to mass transfer, gravitational radiation, and tidal forces between the donor, accretor, and the orbit. The theoretical framework that we have outlined in this paper can be used to generate the models for the rotational angular velocities and the orbital periods of close DWD binaries in general. Thus, we implemented the ballistic mass transfer treatment developed in [19] to calculate the changes to the evolution of orbital parameters during direct impact mass transfer from the donor to the accretor, which can be described by numerical solutions. Here, the numerical solutions that we have presented are more specific than the analytical solutions of the previous studies in the sense that we allow for conservative mass transfer. By implementing this method, we found that the number of stable close DWD binaries increased for both weak and strong tidal coupling compared with the results of [9] and [10], which shown as in Figures 1-10.

A direct comparison of the results obtained from the numerical solutions in Section (3) with those of analytical solutions in Section (4) shows that both the numerical solution and the Eggleton approximation for the Roche lobe calculations are quite accurate.

In many cases the orbital angular momentum lost from the orbit can be significantly less than the standard assumption, making this process less destabilizing than expected. This may allow for more DWD to survive the dynamical instability of mass transfer rate and evolve into systems like AM CVn, instead of merging to create Type Ia supernovae evolving to higher separations and diminishing mass transfer rates. This result, which was not predicted by the analytical solution in [28] has important consequences for population synthesis models of these objects. Furthermore, we have seen that the near constancy of the mass transfer rate over most of the mass transfer phase seen in the results by previous studies is not a generic feature of this type of evolution but rather a consequence of a particular choice of orbital parameters.

In a few cases, we have shown that mass transfer may increase the orbital angular momentum of the orbit, thereby providing a stabilizing effect on the orbit. Hence, any stabilizing effect increases the chances of a long-lived close DWD binaries, lending acceptance to the creation of AM CVn through the DWD models. 


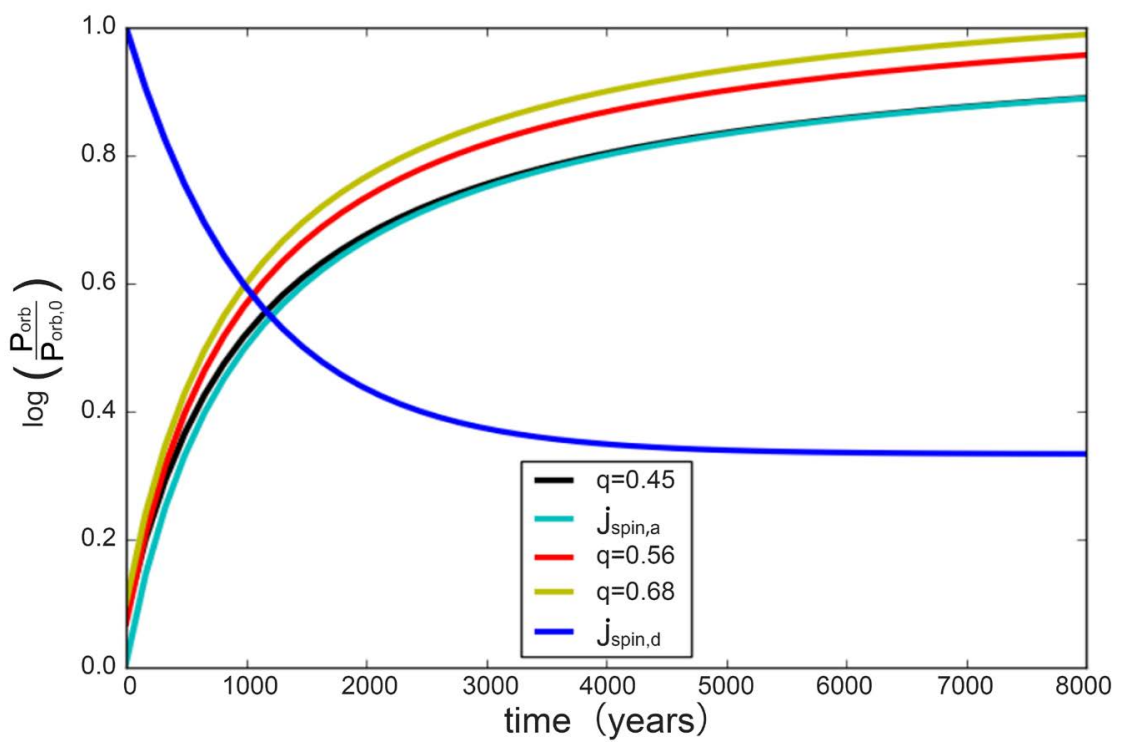

Figure 10. Evolution of the orbital period, $P_{o r b}$, compared to its initial orbital period, $P_{o r b, 0}$, for three different mass ratios with synchronization time scale, $\tau=10^{15}$. Here we observe that the $P_{o r b}$ increases due to the effect of the tides. As a result, $\dot{M}_{d}$ increases rapidly and the accretor spin, $\dot{J}_{\text {spin, }, a}$ increases, whereas the donor spin, $\dot{J}_{\text {spin, } d}$ decreases.

We also account for the modification of the Roche lobe size due to the orbital period and $\zeta_{a d}$ of the donor stars. As a result, we found that the number of stable systems increases, particularly for the case of strong tidal coupling, as shown in Figure 1, Figure 4, Figure 5 and Figure 7. Note that all the systems that undergo direct impact systems, at least for the system orbital parameters and synchronization time scales that we have considered. Thus, when the system is dynamically unstable, the time scale for changes in the mass transfer rate becomes greater than the time scale at which the $P_{o r b}$ increases due to the effect of tides. As a result, the $\dot{M}_{d}$ increases rapidly the spin angular momentum of the accretor increases significantly. We conclude that the stable systems occurred when using the Eggleton approximation for the Roche lobe calculation create artificially high mass transfer rates which leads to an artificially high number of dynamically unstable systems. Hence, our finding yields a higher number of stable systems.

Finally, the large scale numerical computations presented in this paper have provided the realistic models that describing the evolution of angular momentum and orbital period changes and its stability of mass transfer in close DWD binary systems.

\section{Acknowledgements}

We thank Ethiopian Space Science and Technology Institute (ESSTI)-Entoto Observatory and Research Center (EORC), Astronomy and Astrophysics Research Development Department for giving research opportunity and facilities. 
Negu S. H. thanks Jigjiga University for giving study leave. This research has made use of NASA's Astrophysical Data System.

\section{Conflicts of Interest}

The authors declare no conflicts of interest regarding the publication of this paper.

\section{References}

[1] Nelemans, G., Yungelson, L.R. and Portegies Zwart, S.F. (2001) The Gravitational Wave Signal from the Galactic Disk Population of Binaries Containing Two Compact Objects. Astronomy \& Astrophysics, 375, 890-898.

[2] Toonen, S., Nelemans, G. and Portegies Zwart, S. (2012) Supernova Type Ia Progenitors from Merging Double White Dwarfs. Using a New Population Synthesis Model. Astronomy \& Astrophysics, 546, A70.

[3] Fuller, J. and Lai, D. (2012) Dynamical Tides in Compact White Dwarf Binaries: Tidal Synchronization and Dissipation. Monthly Notices of the Royal Astronomical Society, 421, 426.

[4] Burkart, J., Quataert, E., Arras, P. and Weinberg, N.N. (2013) Tidal Resonance Locks in Inspiraling White Dwarf Binaries. Monthly Notices of the Royal Astronomical Society, 433, 332. https://doi.org/10.1093/mnras/stt726

[5] Tutukov, A. and Yungelson, L.R. (1996) Double-Degenerate Semidetached Binaries with Helium Secondaries: Cataclysmic Variables, Supersoft X-Ray Sources, Supernovae and Accretion-Induced Collapses. Monthly Notices of the Royal Astronomical Society, 280, 1035-1045. https://doi.org/10.1093/mnras/280.4.1035

[6] Nelemans, G., Portegies Zwart, S.F., Verbunt, F. and Yungelson, L.R. (2001) Population Synthesis for Double White Dwarfs. II. Semi-Detached Systems: AM CVn Stars. Astronomy \& Astrophysics, 368, 939-949.

[7] Frank, J., King, A. and Raine, D. (2002) Accretion Power in Astrophysics. Cambridge University Press, Cambridge. https://doi.org/10.1017/CBO9781139164245

[8] Sepinsky, J. and Kalogera, V. (2014) Angular Momentum Exchange in White Dwarf Binaries Accreting Through Direct Impact. The Astrophysical Journal, 785, 157 (8 p).

[9] Marsh, T.R., Nelemans, G. and Steeghs, D. (2004) Mass Transfer Between Double White Dwarfs. Monthly Notices of the Royal Astronomical Society, 350, 113-128. https://doi.org/10.1111/j.1365-2966.2004.07564.x

[10] Gokhale, V., Peng, X.M. and Frank, J. (2007) Evolution of Close White Dwarf Binaries. The Astrophysical Journal, 655, 1010-1024. https://doi.org/10.1086/510119

[11] Soberman, G.E., Phinney, E.S. and van den Heuvel, E.P.J. (1997) Stability Criteria for Mass Transfer in Binary Stellar Evolution. Astronomy \& Astrophysics, 327, 620-635.

[12] Negu, S.H. and Tessema, S.B. (2015) Mass Transfer in Binary Stellar Evolution and Its Stabilty. International Journal of Astronomy and Astrophysics, 5, 222-241. https://doi.org/10.4236/ijaa.2015.53026

[13] Verbunt, F. and Rappaport, S. (1988) Mass Transfer Instabilities Due to Angular Momentum Flows in Close Binaries. The Astrophysical Journal, 332, 193-198. https://doi.org/10.1086/166645

[14] Webbink, R.F. and Iben Jr., I. (1987) Tidal Interaction and Coalescence of Close 
Binary White Dwarfs. Proceedings of the 2nd Conference on Faint Blue Stars, 445.

[15] Eggleton, P.P. (1983) Approximations to the Radii of Roche Lobes. The Astrophysical Journal, 268, 368-369. https://doi.org/10.1086/160960

[16] Sepinsky, J., Willems, B. and Kalogera, V. (2007) Equipotential Surfaces and Lagrangian Points in Nonsynchronous, Eccentric Binary and Planetary Systems. The Astrophysical Journal, 660, 1624-1635. https://doi.org/10.1086/513736

[17] Landau, L.D. and Lifshitz, E.M. (1975) The Classical Theory of Fields. Pergamon Press, Oxford.

[18] Campbell, C.G. (1984) Tidal Effects in Twin-Degenerate Binaries. MNRAS, 207, 433-443. https://doi.org/10.1093/mnras/207.3.433

[19] Sepinsky, J., Willems, B., Kalogera, V. and Rasio, A.F. (2010) Interacting Binaries with Eccentric Orbits. III. Orbital Evolution Due to Direct Impact and Self-Accretion. The Astrophysical Journal, 724, 546-558. https://doi.org/10.1088/0004-637X/724/1/546

[20] King, A.R. and Kolb, U. (1995) Consequential Angular Momentum Loss and the Period Gap of Cataclysmic Variables. The Astrophysical Journal, 439, 330-336. https://doi.org/10.1086/175176

[21] Paczynski, B. and Sienkiewicz, R. (1972) Evolution of Close Binaries VIII. Mass Exchange on the Dynamical Time Scale. Acta Astronautica, 22, 73-91.

[22] Webbink, R.F. (1977) The Evolution of Low Mass Close Binary Systems. III-1.50 + 0.50 Solar Masses: Unsteady Mass Loss and Shrinking Secondaries. The Astrophysical Journal, 211, 486-498. https://doi.org/10.1086/154956

[23] Savonije, G.J. (1978) Roche-Lobe Overflow in X-Ray Binaries. Astronomy \& Astrophysics, 62, 317-338.

[24] Webbink, R.F. (1984) Double White Dwarfs as Progenitors of R Coronae Borealis Stars and Type I Supernovae. The Astrophysical Journal, 277, 355-360. https://doi.org/10.1086/161701

[25] Chandrasekhar, S. (1967) An Introduction to the Study of Stellar Structure. Dover, New York.

[26] Paczynski, B. (1971) Evolutionary Processes in Close Binary Systems. Annual Review of Astronomy and Astrophysics, 9, 183. https://doi.org/10.1146/annurev.aa.09.090171.001151

[27] D’Antona, F., Ventura, P., Burderi, L. and Teodorescu, A. (2006) Modeling the Closest Double Degenerate System RX J0806.3+1527 and Its Decreasing Period. The Astrophysical Journal, 653, 1429. https://doi.org/10.1086/507408

[28] Hjellming, M.S. and Webbink, R.F. (1987) Thresholds for Rapid Mass Transfer in Binary Systems. The Astrophysical Journal, 318, 794-808.

https://doi.org/10.1086/165412

[29] Galassi, M.J.D., Theiler, J., Gough, B., Jungman, G., Booth, M. and Rossi, F. (2006) GNU Scientific Library Reference Manual. 2nd Edition, Network Theory Ltd.

[30] Lubow, S.H. and Shu, F.H. (1975) Gas Dynamics of Semidetached Binaries. The Astrophysical Journal, 198, 383-405. https://doi.org/10.1086/153614 